Algebraic $\& \mathcal{G}$ Geometric Topology

Volume 2 (2002) 11-32

Published: 15 January 2002

ATG

\title{
Homfly polynomials of generalized Hopf links
}

\author{
Hugh R. Morton \\ RICHARD J. HADJI
}

\begin{abstract}
Following the recent work by T.-H. Chan in [3] on reverse string parallels of the Hopf link we give an alternative approach to finding the Homfly polynomials of these links, based on the Homfly skein of the annulus. We establish that two natural skein maps have distinct eigenvalues, answering a question raised by Chan, and use this result to calculate the Homfly polynomial of some more general reverse string satellites of the Hopf link.
\end{abstract}

AMS Classification $57 \mathrm{M} 25$

Keywords Hopf link, satellites, reverse parallels, Homfly polynomial

\section{Introduction}

In [3] T.-H. Chan discusses the Homfly polynomial of reverse string parallels $H\left(k_{1}, k_{2} ; n_{1}, n_{2}\right)$ of the Hopf link. In this paper we analyse their structure more closely using the Homfly skein of the annulus and identify the eigenvalues and eigenvectors which occur naturally in this approach.

This allows us to readily calculate the Homfly polynomial of satellites of the Hopf link which consist of a reverse string parallel around one component combined with a completely general reverse string decoration on the other.

\section{The Homfly polynomial}

Various versions of the Homfly polynomial appear in the literature. The framed version to the fore in this paper is defined by the following skein relations:

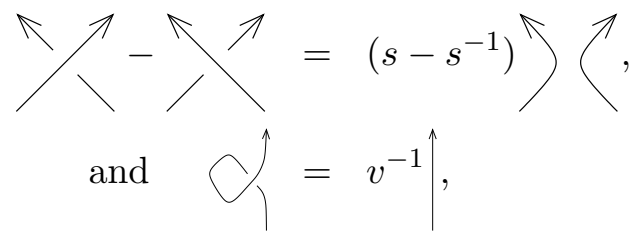


with the Homfly polynomial of the empty diagram being normalized to 1 , and that of the null-homotopic loop therefore being $\delta=\frac{v^{-1}-v}{s-s^{-1}}$. There is a discussion of isomorphic variants of these skein relations given in [2] and [7].

Notation For a link $L$, we denote the evaluation of its Homfly polynomial by $P(L)$.

Remark (i) The Homfly polynomial of the $m$-component unlink, $\mathcal{U}^{m}=$ $\sqcup_{i=1}^{m} \bigcirc$, is $P\left(\mathcal{U}^{m}\right)=\delta^{m}$.

(ii) If $L^{*}$ is the reflection of a link $L$, then

$$
P\left(L^{*}\right)(s, v)=P(L)\left(s^{-1}, v^{-1}\right) .
$$

\section{Diagrams in the annulus}

We shall now introduce the basic idea of diagrams in the annulus. Given the annulus $F=S^{1} \times I$, a diagram in $F$ consists of closed curves (as with a standard knot diagram) with a finite number of crossing points. At a crossing point the strands are distinguished in the conventional way as an over-crossing and an under-crossing.

\section{Satellites of Hopf links}

The Hopf link is the simplest non-trivial link involving just two unknots linked together. When giving this link orientation, two distinct links are formed. We shall call these $H_{+}$and $H_{-}$, as shown in Figure 1 . The Homfly polynomial can
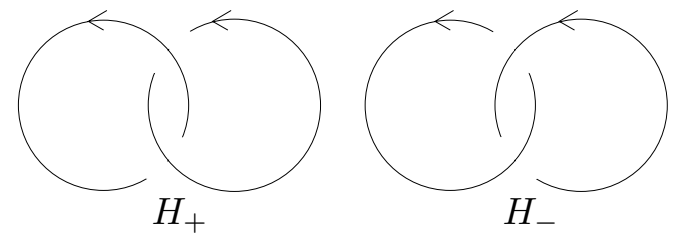

Figure 1: The links $H_{+}$and $H_{-}$

then be calculated using the above skein relations. We have that:

$$
\begin{aligned}
P\left(H_{+}\right) & =\left(\frac{v^{-1}-v}{s-s^{-1}}\right)^{2}+v^{-2}-1 ; \\
\text { and } P\left(H_{-}\right) & =\left(\frac{v^{-1}-v}{s-s^{-1}}\right)^{2}+v^{2}-1 .
\end{aligned}
$$

Algebraic 83 Geometric Topology, Volume 2 (2002) 
We now use $H_{+}$and $H_{-}$as starting points for the construction of satellite links. We do this by considering the two components of the Hopf links and decorating them. For example, take $P_{1}$ and $P_{2}$ as diagrams in the annulus. Now starting with $H_{+}$we decorate its two components with $P_{1}$ and $P_{2}$ respectively, obtaining the link $H_{+}\left(P_{1}, P_{2}\right)$, as shown in Figure 2. Now clearly $H_{+}\left(P_{1}, P_{2}\right)$ and $H_{+}\left(P_{2}, P_{1}\right)$ are equivalent links. An analogous construction is possible for $H_{-}$.

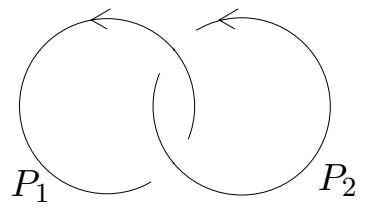

Figure 2: The link $H_{+}\left(P_{1}, P_{2}\right)$

With such a construction, a great variety of links may be realised. In particular, the generalized Hopf links which are the topic of [3] can be constructed. For example, if we take $P_{1}$ and $P_{2}$ as shown in Figure 3 , then $H_{+}\left(P_{1}, P_{2}\right)$ is the

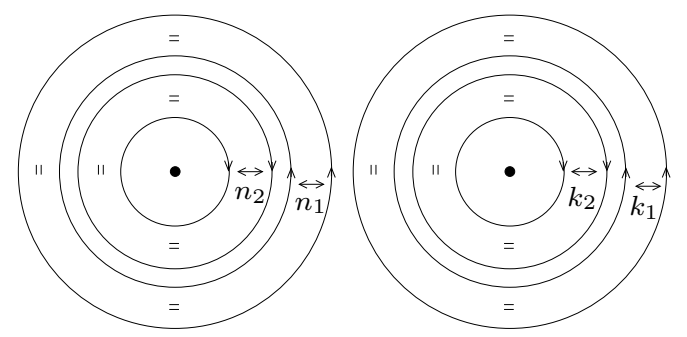

Figure 3: The diagrams $P_{1}$ and $P_{2}$

link Chan refers to as $H\left(k_{1}, k_{2} ; n_{1}, n_{2}\right)$, as shown in Figure 4.

With such links in mind, we make the following observation:

Observation The links

$$
H\left(k_{1}, k_{2} ; n_{1}, n_{2}\right), H\left(n_{1}, n_{2} ; k_{1}, k_{2}\right), H\left(k_{2}, k_{1} ; n_{2}, n_{1}\right), H\left(n_{2}, n_{1} ; k_{2}, k_{1}\right),
$$

and

$$
H^{*}\left(k_{2}, k_{1} ; n_{1}, n_{2}\right), H^{*}\left(n_{1}, n_{2} ; k_{2}, k_{1}\right), H^{*}\left(k_{1}, k_{2} ; n_{2}, n_{1}\right), H\left(n_{2}, n_{1} ; k_{1}, k_{2}\right),
$$

are all equivalent links. 


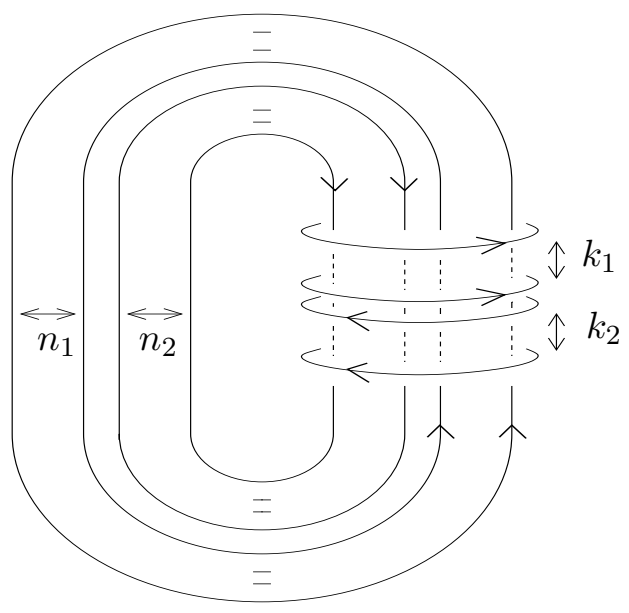

Figure 4: The generalized Hopf link $H\left(k_{1}, k_{2} ; n_{1}, n_{2}\right)$

\section{The skein of the annulus}

We have introduced the concept of having diagrams in the annulus, and used this to construct satellites of the Hopf links. We now describe the skein of the annulus, denoted by $\mathcal{C}$.

The Homfly skein of the annulus $\mathcal{C}$, as discussed in [8] and originally in the preprint of [12] in 1988, is defined as linear combinations of diagrams in the annulus, modulo the Homfly skein relations given above in the Introduction. We shall represent an element $X \in \mathcal{C}$ diagramatically as in Figure 5.

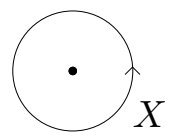

Figure 5: An element $X \in \mathcal{C}$

The skein $\mathcal{C}$ has a product induced by placing one annulus outside another. This defines a bilinear product $\mathcal{C} \times \mathcal{C} \rightarrow \mathcal{C}$, under which $\mathcal{C}$ becomes an algebra. This algebra is clearly commutative (lift the inner annulus up and stretch it so that the outer one will fit inside it).

Turaev [12] showed that $\mathcal{C}$ is freely generated as an algebra by $\left\{A_{m}, m \in \mathbb{Z}\right\}$ where $A_{m}$ is the skein element shown in Figure 6 and the sign of the index $m$ indicates the orientation of the curve. A positive $m$ denotes counter-clockwise 
orientation and a negative $m$ clockwise orientation. The element $A_{0}$ is the identity element, represented by the empty diagram.

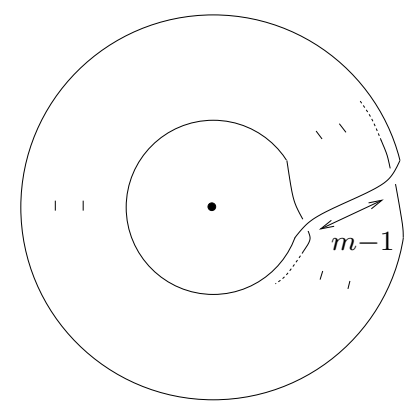

Figure 6: An element $A_{m} \in \mathcal{C}$, for $m \in \mathbb{Z}$

We now define two natural linear maps, $\varphi$ and $\bar{\varphi}$, on the skein of the annulus in the following way:

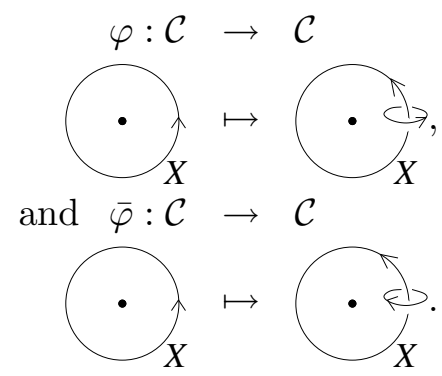

These two maps can be related via a map $\rho: \mathcal{C} \rightarrow \mathcal{C}$ which takes the annulus and its contents and flips it over. Clearly $\rho^{-1}=\rho$. It is then clear that $\bar{\varphi}=\rho^{-1} \varphi \rho=\rho \varphi \rho$. However, this does not mean that the maps $\varphi$ and $\bar{\varphi}$ are conjugate as this does not define an inner automorphism of $\mathcal{C}$.

Now consider the satellites of Hopf links discussed earlier in the Introduction as elements of the skein $\mathcal{C}$. We can then use compositions of the maps $\varphi$ and $\bar{\varphi}$ to construct a subset of such links. In particular, for the element $A=A_{1}^{n_{1}} A_{-1}^{n_{2}} \in \mathcal{C}$, we have:

$$
H\left(k_{1}, k_{2} ; n_{1}, n_{2}\right)=\varphi^{k_{1}}\left(\bar{\varphi}^{k_{2}}(A)\right) .
$$

It will therefore aid our investigation of the $H\left(k_{1}, k_{2} ; n_{1}, n_{2}\right)$ and their Homfly polynomial if we were to look more closely at the maps $\varphi$ and $\bar{\varphi}$, in particular at their eigenvalues. This will be achieved through considering certain subspaces of $\mathcal{C}$ and the restrictions of the maps $\varphi$ and $\bar{\varphi}$ to these subspaces. 


\section{Subspaces of $\mathcal{C}$}

The algebra $\mathcal{C}$ can be thought of as the product of subalgebras $\mathcal{C}^{+}$and $\mathcal{C}^{-}$ which are generated by $\left\{A_{m}: m \in \mathbb{Z}, m \geq 0\right\}$ and $\left\{A_{m}: m \in \mathbb{Z}, m \leq 0\right\}$ respectively.

Remark In his thesis, [6], Lukac shows how to calculate the Homfly polynomial of any satellite of the Hopf link, when the decorations are chosen from $\mathcal{C}^{+}$. Here we consider the full skein $\mathcal{C}$, allowing more general reverse string decorations.

\subsection{The subspace $\mathcal{C}^{(n)} \subset \mathcal{C}^{+}$}

Given linear combinations of oriented $n$-tangles as shown in Figure 7 , modulo

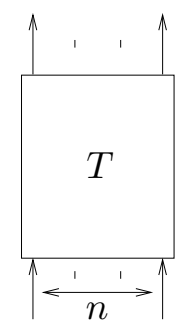

Figure 7: An oriented $n$-tangle

the Homfly skein relations, we can form an algebra. Multiplication within this algebra is induced by stacking one tangle on top of another.

Now take the well-known Hecke algebra $H_{n}$ of type $A_{n-1}$. It has several different incarnations, but is most conveniently thought of in this context as having explicit presentation:

$$
H_{n}=\left\langle\begin{array}{l|l}
\sigma_{i}: i=1, \ldots, n-1 \mid \begin{array}{l}
\sigma_{i} \sigma_{j}=\sigma_{j} \sigma_{i}:|i-j|>1 \\
\sigma_{i} \sigma_{i+1} \sigma_{i}=\sigma_{i+1} \sigma_{i} \sigma_{i+1}: 1 \leq i<n-1 ; \\
\sigma_{i}-\sigma_{i}^{-1}=z
\end{array}
\end{array}\right\rangle .
$$

It is shown in [9] that $H_{n}$, with $z=s-s^{-1}$ and coefficient ring extended to include $v^{ \pm 1}$ and $s^{ \pm 1}$, is isomorphic to the skein theoretic algebra described above. In this algebra the extra variable $v$ in the coefficient ring allows us to reduce general tangles to linear combinations of braids, by means of the skein 


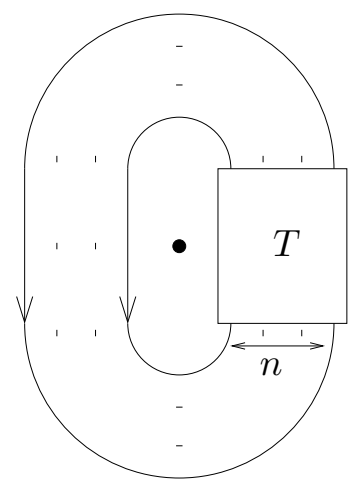

Figure 8: An element of the subspace $\mathcal{C}^{(n)}$

relations in the introduction. The variable $v$ comes into play in dealing with curls using the second skein relation and in handling disjoint closed curves.

Wiring these $n$-tangles into the annulus as shown in Figure 8 gives a linear subspace of $\mathcal{C}^{+}$which we shall call $\mathcal{C}^{(n)}$. This subspace is the image of $H_{n}$ under the closure map $\wedge: H_{n} \rightarrow \mathcal{C}^{(n)}$. For an $n$-tangle $T \in H_{n}$, we denote its image under the closure map $\wedge(T)$ or $\hat{T}$.

The subspace $\mathcal{C}^{(n)}$ is then spanned by monomials in $\left\{A_{m}\right\}$, with $m \in \mathbb{Z}^{+}$, of total weight $n$, where $\operatorname{wt}\left(A_{m}\right)=m$. It is clear that this spanning set consists of $\pi(n)$ elements, the number of partitions of $n$. (The standard notation used for the number of partitions of an integer $n$ is $p(n)$; our alternative has been chosen to avoid a clash with notation required later in this paper.) $\mathcal{C}^{+}$is then graded as an algebra:

$$
\mathcal{C}^{+}=\bigoplus_{n=0}^{\infty} \mathcal{C}^{(n)}
$$

Now due to the relationship between $H_{n}$ and $\mathcal{C}^{(n)}$ it will be useful here to recall some well-established facts about $H_{n}$. In particular, we shall concentrate on facts about certain elements in $H_{n}$.

Firstly, there is a set of quasi-idempotent elements of $H_{n}$ discussed first in [4] and given a geometric interpretation in [1] (see also [2]). We shall denote these elements $e_{\lambda}$, one for each partition $\lambda$ of $n$, with $\emptyset$ denoting the unique partition of 0 .

Now, given the element $T^{(n)} \in H_{n}$ shown in Figure 9, one can use skein theoretic techniques to prove the following corollary of Theorem 19 in [2] (see [7]), 


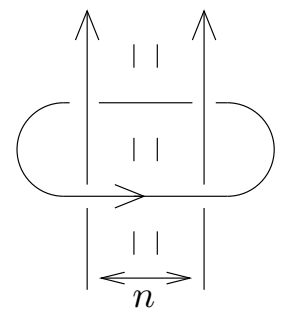

Figure 9: The $n$-tangle $T^{(n)}$

Corollary (of Theorem 19, [2]) $T^{(n)} e_{\lambda}=t_{\lambda} e_{\lambda}$ where

$$
t_{\lambda}=\left(s-s^{-1}\right) v^{-1} \sum_{\substack{\text { cells } \\ \text { in } \lambda}} s^{2(\text { content })}+\delta .
$$

Moreover, the scalars $t_{\lambda}$ are different for each partition $\lambda$.

Reverse the orientation of the encircling string in $T^{(n)}$ and call this $\bar{T}^{(n)}$. Then, using similar techniques, one can show

Lemma $1 \quad \bar{T}^{(n)} e_{\lambda}=\bar{t}_{\lambda} e_{\lambda}$ where

$$
\bar{t}_{\lambda}=-\left(s-s^{-1}\right) v \sum_{\substack{\text { cells } \\ \text { in } \lambda}} s^{-2(\text { content })}+\delta .
$$

Moreover, the scalars $\bar{t}_{\lambda}$ are also different for each partition $\lambda$.

Remark An alternative proof to this lemma could be made using the natural skein mirror map $-: \mathcal{C}^{(n)} \rightarrow \mathcal{C}^{(n)}$. This map switches all crossings in a tangle and inverts the scalars $v$ and $s$ in the coefficient ring. Clearly this map can be seen to leave the skein relations unchanged. One must then note that the $e_{\lambda}$ are invariant under this map and that $-\left(T^{(n)}\right)=\bar{T}^{(n)}$. We then apply these facts to the above Corollary and the result follows immediately.

We now link these facts to the maps $\varphi$ and $\bar{\varphi}$, through use of the closure map. Take an element $S \in H_{n}$ with $\hat{S} \in \mathcal{C}^{(n)}$ and compose it with $T^{(n)}$. Then $\wedge\left(S T^{(n)}\right)=\varphi(\hat{S})$. Similarly $\wedge\left(S \bar{T}^{(n)}\right)=\bar{\varphi}(\hat{S})$.

The restrictions $\left.\varphi\right|_{\mathcal{C}^{(n)}}$ and $\left.\bar{\varphi}\right|_{\mathcal{C}^{(n)}}$ clearly carry $\mathcal{C}^{(n)}$ to itself.

Theorem 2 [7] The eigenvalues of $\left.\varphi\right|_{\mathcal{C}^{(n)}}$ are all distinct as are the eigenvalues of $\left.\bar{\varphi}\right|_{\mathcal{C}^{(n)}}$. 
Proof We prove the first statement with the second following in exactly the same way.

Set $Q_{\lambda}=\hat{e}_{\lambda} \in \mathcal{C}^{(n)}$. Then the closure of $T^{(n)} e_{\lambda}$ is $\varphi\left(Q_{\lambda}\right)$. However, $T^{(n)} e_{\lambda}=$ $t_{\lambda} e_{\lambda}$, hence $\varphi\left(Q_{\lambda}\right)=t_{\lambda} Q_{\lambda}$. The element $Q_{\lambda}$ is then an eigenvector of $\varphi$ with eigenvalue $t_{\lambda}$. There are $\pi(n)$ of these eigenvectors, and the eigenvalues are all distinct by [2]. Since $\mathcal{C}^{(n)}$ is spanned by $\pi(n)$ elements we can deduce that the elements $Q_{\lambda}$ form a basis for $\mathcal{C}^{(n)}$ and that the eigenspaces are all 1-dimensional.

This proof is quite instructive as it establishes that the $Q_{\lambda}$ with $|\lambda|=n$ are a basis for $\mathcal{C}^{(n)}$. Hence any element in $\mathcal{C}^{(n)}$ can be written as a linear combination of the $Q_{\lambda}$ with $|\lambda|=n$. It also follows that any element of $\mathcal{C}^{(n)}$ which is an eigenvector of $\varphi$ (and similarly $\bar{\varphi}$ ) must be a multiple of some $Q_{\lambda}$. Finally, we notice that the eigenvalues of the $\varphi$ and $\bar{\varphi}$ are the $t_{\lambda}$ and $\bar{t}_{\lambda}$ we found earlier.

\subsection{The subspace $\mathcal{C}^{(n, p)}$}

We now extend our view of the skein of the annulus to include strings oriented in both directions. We do this through considering the closure of oriented $(n, p)$-tangles such as the one shown in Figure 10. We denote the algebra

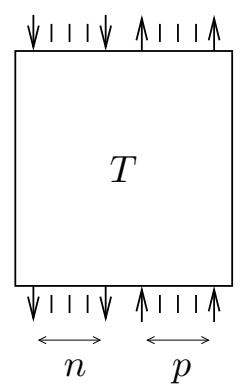

Figure 10: An oriented $(n, p)$-tangle

formed through considering linear combinations of such $(n, p)$-tangles, modulo the Homfly skein relations, by $M_{n, p}$. For further information on $M_{n, p}$ see [10] or [5]. The image of $M_{n, p}$ under the closure map shall be denoted $\mathcal{C}^{(n, p)} \subset \mathcal{C}$.

Unlike the case for $\mathcal{C}^{(n)}$ where $\mathcal{C}^{(n)} \cap \mathcal{C}^{(n-1)}=\emptyset$, we have that:

$$
\mathcal{C}^{(n, p)} \supset \mathcal{C}^{(n-1, p-1)} \supset \mathcal{C}^{(n-2, p-2)} \supset \cdots \supset \begin{cases}\mathcal{C}^{(n-p, 0)} & \text { if } \min (n, p)=p, \\ \mathcal{C}^{(0, p-n)} & \text { if } \min (n, p)=n,\end{cases}
$$


however, it should be noted that for each $\mathcal{C}^{(i, j)}$ in the sequence, the difference $i-j$ remains constant. Also,

$$
\begin{aligned}
\mathcal{C}^{(m, 0)} & \cong \mathcal{C}_{(-)}^{(m)} \\
\text { and } \quad \mathcal{C}^{(0, m)} & \cong \mathcal{C}_{(+)}^{(m)},
\end{aligned}
$$

where the subscripts indicate the direction of the strings around the centre of the annulus. However, we do have that $\mathcal{C}^{\left(n_{1}, p_{1}\right)} \cap \mathcal{C}^{\left(n_{2}, p_{2}\right)}=\emptyset$ if $n_{1}-p_{1} \neq n_{2}-p_{2}$. We find that $\mathcal{C}^{(n, p)}$ is spanned by suitably weighted monomials in

$$
\left\{A_{-n}, \ldots, A_{-1}, A_{0}, A_{1}, \ldots, A_{p}\right\} .
$$

We can then see that:

$$
\mathcal{C}^{(n, p)}=\left(\mathcal{C}_{(-)}^{(n)} \times \mathcal{C}_{(+)}^{(p)}\right)+\mathcal{C}^{(n-1, p-1)} .
$$

The spanning set of $\mathcal{C}^{(n, p)}$ consists of $\pi(n, p)$ elements, where

$$
\begin{aligned}
\pi(n, p) & :=\sum_{j=0}^{k} \pi(n-j) \pi(p-j) \\
& =\pi(n) \pi(p)+\cdots+\pi(n-k) \pi(p-k),
\end{aligned}
$$

with $k=\min (n, p)$.

Similar to the grading of $\mathcal{C}^{+}$with the $\mathcal{C}^{(n)}$ we can think of the whole of $\mathcal{C}$ in terms of the $\mathcal{C}^{(n, p)}$ :

$$
\mathcal{C}=\bigoplus_{k=-\infty}^{\infty}\left(\bigcup_{n, p \geq 0}\left\{\mathcal{C}^{(n, p)}: n-p=k\right\}\right)
$$

We now use an example to illustrate what we meant by "suitably weighted" monomials in the $A_{i}$.

Example Consider when $n=3$ and $p=2$. The spanning set of $\mathcal{C}^{(3,2)}$ consists of $9(=3 \cdot 2+2 \cdot 1+1 \cdot 1)$ elements, since

$$
\mathcal{C}^{(3,2)}=\left(\mathcal{C}_{(-)}^{(3)} \times \mathcal{C}_{(+)}^{(2)}\right)+\left(\mathcal{C}_{(-)}^{(2)} \times \mathcal{C}_{(+)}^{(1)}\right)+\left(\mathcal{C}_{(-)}^{(1)} \times \mathcal{C}_{(+)}^{(0)}\right)
$$

The spanning set is therefore:

$$
\begin{array}{r}
\left\{A_{-3} A_{2}, A_{-3} A_{1}^{2}, A_{-2} A_{-1} A_{2}, A_{-2} A_{-1} A_{1}^{2}, A_{-1}^{3} A_{2}, A_{-1}^{3} A_{1}^{2}, A_{-2} A_{1},\right. \\
\left.A_{-1}^{2} A_{1}, A_{-1}\right\},
\end{array}
$$

where, for example, the element $A_{-2} A_{1}$ is obtained from closing an element in $M_{3,2}$ as shown in Figure 11.

Algebraic $6 \mathcal{G}$ Geometric $\mathcal{T}$ opology, Volume 2 (2002) 


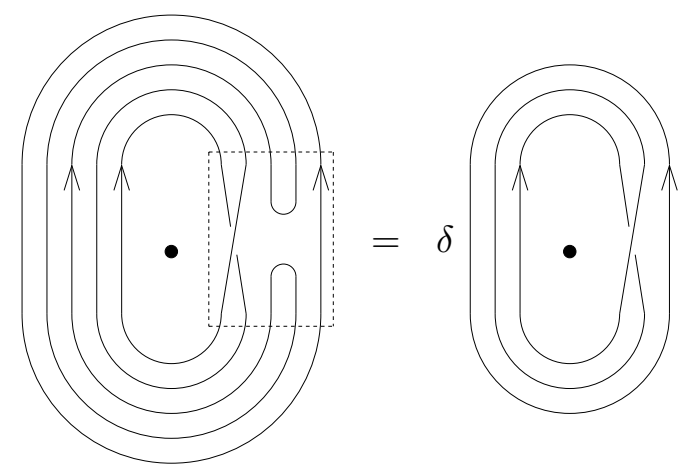

Figure 11: The generator $A_{-2} A_{1}$

Following an exactly analogous procedure in $M_{n, p}$ as in $H_{n}$, we define central elements $T^{(n, p)}$ and $\bar{T}^{(n, p)}$ by diagrams similar to $T^{(n)}$ and $\bar{T}^{(n)}$ respectively as in Figure 12.

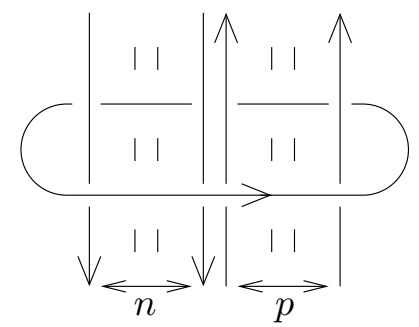

Figure 12: The $(n, p)$-tangle $T^{(n, p)}$

Definition 1 (see [10],[5]) Let $M_{n, p}^{(i)}$ denote the sub-algebra of $M_{n, p}$ spanned by elements with "at least" $i$ pairs of strings turning back.

Remark (i) An $(n, p)$-tangle is said to have "at least" $l$ pairs of strings which turn back if it can be written as a product $T_{1} T_{2}$ of an $\{(n, p),(n-l, p-l)\}$-tangle $T_{1}$ and an $\{(n-l, p-l),(n, p)\}$-tangle $T_{2}$ as illustrated in Figure 13.

(ii) The $M_{n, p}^{(i)}$ are two-sided ideals and there is a filtration:

$$
M_{n, p} \cong M_{n, p}^{(0)} \triangleright M_{n, p}^{(1)} \triangleright \cdots \triangleright M_{n, p}^{(k)},
$$

where $k=\min (n, p)$. 


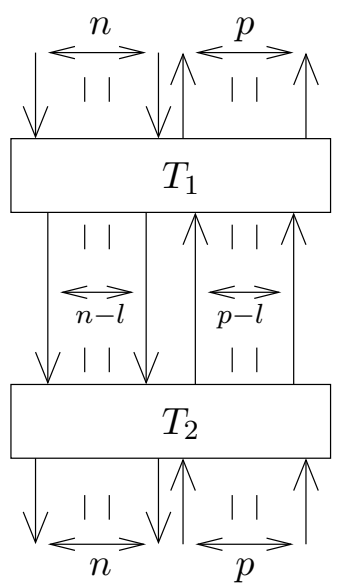

Figure 13: A tangle with at least $l$ pairs of strings which turn back

\section{Lemma 3}

$$
\begin{aligned}
& T^{(n, p)}=T^{(n, p) \prime}+w, \\
& \bar{T}^{(n, p)}=\bar{T}^{(n, p) \prime}+\bar{w}
\end{aligned}
$$

where

$$
\begin{aligned}
& T^{(n, p) \prime}=T_{(-)}^{(n)} \otimes 1_{(+)}^{(p)}+1_{(-)}^{(n)} \otimes T_{(+)}^{(p)}-\delta 1_{(-)}^{(n)} \otimes 1_{(+)}^{(p)}, \\
& \bar{T}^{(n, p) \prime}=\bar{T}_{(-)}^{(n)} \otimes 1_{(+)}^{(p)}+1_{(-)}^{(n)} \otimes \bar{T}_{(+)}^{(p)}-\delta 1_{(-)}^{(n)} \otimes 1_{(+)}^{(p)},
\end{aligned}
$$

and $w, \bar{w} \in M_{n, p}^{(1)}$.

Notation The tensor product $S \otimes T$ indicates the juxtaposition of tangles $S$ and $T$.

Proof (of Lemma 3) We prove the result for $T^{(n, p)}$, with the result for $\bar{T}^{(n, p)}$ following in exactly the same way. Throughout this proof, we use a standard notation setting $s-s^{-1}=z$.

We first define some elements in $M_{n, p}$ represented by tangles as shown in Figure 14.

Now applying the skein relation once to $T^{(n, p)}$ we obtain:

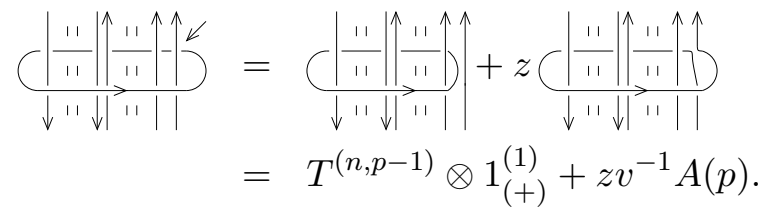

Algebraic 63 Geometric Topology, Volume 2 (2002) 


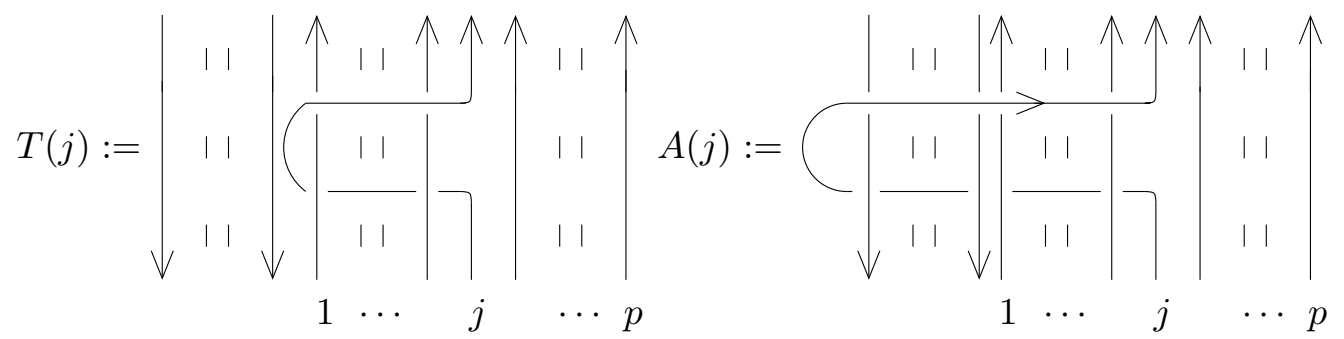

Figure 14: The tangles representing the elements $T(j)$ and $A(j)$ for $1 \leq j \leq p$

Repeated application of the skein relation in this way will clearly yield:

$$
\begin{aligned}
T^{(n, p)} & =T^{(n, 0)} \otimes 1_{(+)}^{(p)}+z v^{-1} \sum_{j=1}^{p} A(j) \\
& =T_{(-)}^{(n)} \otimes 1_{(+)}^{(p)}+z v^{-1} \sum_{j=1}^{p} A(j) .
\end{aligned}
$$

Now observe, similar to a result in [7], we can find:

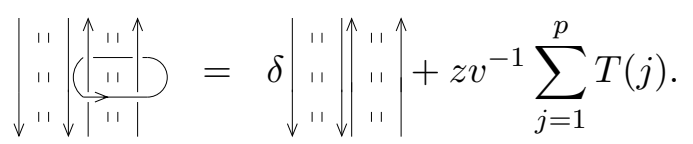

Combining equations 1 and 2 , we see that we are only left to show that:

$$
z v^{-1} \sum_{j=1}^{p} A(j)=z v^{-1} \sum_{j=1}^{p} T(j)+w,
$$

for $w \in M_{n, p}^{(1)}$.

Let $w=\sum_{j=1}^{p} w(j)$. We must now show that for each $j$, with $1 \leq j \leq p$, there exists a $w(j)$ such that:

$$
z v^{-1} A(j)=z v^{-1} T(j)+w(j)
$$


Now,

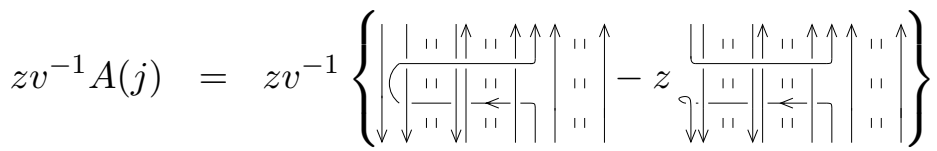

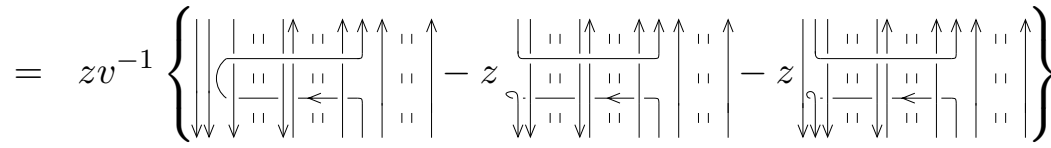

$$
\begin{aligned}
& =\cdots \text { (repeating application of the skein relation) }
\end{aligned}
$$

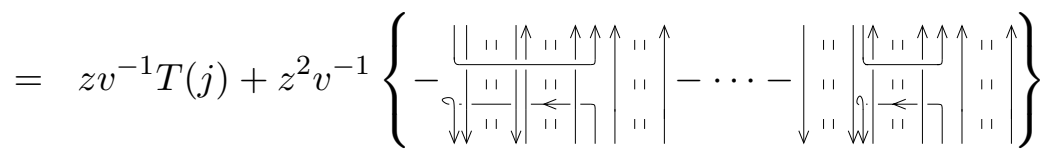

$$
\begin{aligned}
& =z v^{-1} T(j)+w(j) .
\end{aligned}
$$

with $w(j) \in M_{n, p}^{(1)}$.

The result follows.

We can find an obvious set of quasi-idempotent elements in $M_{n, p}$ given by $e_{\lambda, \mu}^{\prime}:=e_{\lambda}^{(-)} \otimes e_{\mu}^{(+)}$formed by the juxtaposition of the Gyoja-Aiston idempotents with appropriate orientations and $|\lambda|=n$ and $|\mu|=p$. There are then $\pi(n) \times$ $\pi(p)$ of these.

We can then use the information in the previous section combined with Lemma 3 to prove the following proposition.

\section{Proposition 4}

$$
\begin{aligned}
T^{(n, p)} e_{\lambda, \mu}^{\prime} & =t_{\lambda, \mu} e_{\lambda, \mu}^{\prime}+w e_{\lambda, \mu}^{\prime} \\
\text { and } \quad \bar{T}^{(n, p)} e_{\lambda, \mu}^{\prime} & =\bar{t}_{\lambda, \mu} e_{\lambda, \mu}^{\prime}+w^{\prime} e_{\lambda, \mu}^{\prime},
\end{aligned}
$$

where,

$$
t_{\lambda, \mu}=\left(s-s^{-1}\right)\left(-v \sum_{\substack{\text { cells } \\ \text { in } \lambda}} s^{-2(\text { content })}+v^{-1} \sum_{\substack{\text { cells } s \\ \text { in } \mu}} s^{2(\text { content })}\right)+\delta
$$

and

$$
\bar{t}_{\lambda, \mu}=\left(s-s^{-1}\right)\left(v^{-1} \sum_{\substack{\text { cells } \\ \text { in } \lambda}} s^{2(\text { content })}-v \sum_{\substack{\text { cells } \\ \text { in } \mu}} s^{-2(\text { content })}\right)+\delta .
$$

Algebraic 83 Geometric Topology, Volume 2 (2002) 
Here we had fixed $|\lambda|$ and $|\mu|$ with values $n$ and $p$ respectively. In fact, we find that $t_{\lambda, \mu}$ and $\bar{t}_{\lambda, \mu}$ have the following property:

Lemma 5 As $\lambda$ and $\mu$ vary over all choices of Young diagram, the values of $t_{\lambda, \mu}$ are all distinct; as are the values of $\bar{t}_{\lambda, \mu}$.

Remark An equivalent way of stating Lemma 5 is that if $t_{\lambda, \mu}=t_{\lambda^{\prime}, \mu^{\prime}}$ then $\lambda=\lambda^{\prime}$ and $\mu=\mu^{\prime}$ (similarly for the $\bar{t}_{\lambda, \mu}$ ).

Proof (of Lemma 5) We prove the first part of the lemma and note that the second part follows immediately due to the observation that $\bar{t}_{\lambda, \mu}=t_{\mu, \lambda}$.

Given $f(s, v)=t_{\lambda, \mu}$ we now show how to recover the Young diagrams $\lambda$ and $\mu$.

From the formula for $t_{\lambda, \mu}$ in Lemma 4 we see that $f(s, v)-\delta$ is a Laurent polynomial in $s$ and $v$, and must be of the form:

$$
\left(s-s^{-1}\right)\left(-v P(s)+v^{-1} Q(s)\right) .
$$

Now consider $P(s)$ and $Q(s)$ individually. It is clear that these are also Laurent polynomials, this time only in the variable $s$. We have

$$
\begin{aligned}
P(s) & =\sum a_{i} s^{-2 i} \\
\text { and } \quad Q(s) & =\sum b_{j} s^{2 j},
\end{aligned}
$$

where $a_{i}$ is the number of cells in $\lambda$ with content $i$, and similarly, $b_{j}$ is the number of cells in $\mu$ with content $j$. Hence we can uniquely construct $\lambda$ and $\mu$.

Now let us return to the maps $\varphi$ and $\bar{\varphi}$, restricting them to the skein $\mathcal{C}^{(n, p)}$.

Theorem 6 The $t_{\lambda, \mu}$ and $\bar{t}_{\lambda, \mu}$ are eigenvalues of $\left.\varphi\right|_{\mathcal{C}^{(n, p)}}$ and $\left.\bar{\varphi}\right|_{\mathcal{C}^{(n, p)}}$ respectively. Moreover, they occur with multiplicity 1.

Proof We prove the result for the $t_{\lambda, \mu}$ with an identical argument proving the result for the $\bar{t}_{\lambda, \mu}$.

Fix an integer $k$ such that $k=p-n$ and $k \geq 0$ (in other words $p \geq n$ - the case for $p<n$ is identical). Write $\mathcal{C}^{(n, p)}$ as $\mathcal{C}^{(n, k+n)}$ and do induction on $n$.

For $n=0$ we have that $\mathcal{C}^{(0, k)} \cong \mathcal{C}^{(k)}$. Now for $|\lambda|=0$ and $|\mu|=k$ we have that $t_{\lambda, \mu}=t_{\mu}$. Moreover, in the proof of Theorem 2 we saw that the $t_{\mu}$ with 
$|\mu|=k$ are eigenvalues of $\left.\varphi\right|_{\mathcal{C}^{(k)}}$. Now since $\mathcal{C}^{(k)} \cong \mathcal{C}^{(0, k)} \subset \mathcal{C}^{(n, k+n)}$ for all $n$, the $t_{\mu}$ are also eigenvalues of $\left.\varphi\right|_{\mathcal{C}^{(n, k+n)}}$.

Now assume that for $|\lambda|<n$ and $|\mu|<k+n$ the $t_{\lambda, \mu}$ are eigenvalues of

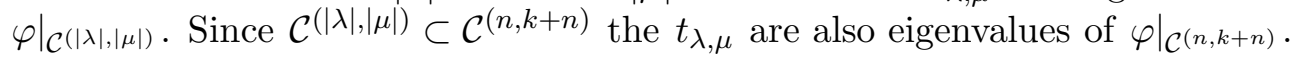

Consider the $t_{\lambda, \mu}$ with $|\lambda|=n$ and $|\mu|=k+n$. By the inductive hypothesis these $t_{\lambda, \mu}$ are not eigenvalues of $\left.\varphi\right|_{\mathcal{C}^{(n-1, k+n-1)}}$ since we have $\pi(n-1, k+n-1)$ eigenvalues and $\mathcal{C}^{(n-1, k+n-1)}$ is spanned by $\pi(n-1, k+n-1)$ elements and by Lemma 5 we have that if $t_{\lambda, \mu}=t_{\lambda^{\prime}, \mu^{\prime}}$ then $\lambda=\lambda^{\prime}$ and $\mu=\mu^{\prime}$.

Define elements $Q_{\lambda, \mu}^{\prime}:=Q_{\lambda}^{(-)} \cdot Q_{\mu}^{(+)}\left(=\wedge\left(e_{\lambda, \mu}^{\prime}\right)\right)$ with $|\lambda|=n$ and $|\mu|=k+n$. Clearly $Q_{\lambda, \mu}^{\prime} \in \mathcal{C}^{(n, k+n)}$.

Now by Lemma 4 ,

$$
\left.\varphi\right|_{\mathcal{C}^{(n, k+n)}}\left(Q_{\lambda, \mu}^{\prime}\right)=t_{\lambda, \mu} Q_{\lambda, \mu}^{\prime}+w^{\prime}
$$

where $w^{\prime} \in \mathcal{C}^{(n-1, k+n-1)}$.

We can find a $v \in \mathcal{C}^{(n-1, k+n-1)}$ such that $\left(\left.\varphi\right|_{\mathcal{C}^{(n, k+n)}}-t_{\lambda, \mu} I\right)(v)=w^{\prime}$.

Now consider $Q_{\lambda, \mu}^{\prime}-v$. This is clearly non-zero. We find:

$$
\begin{aligned}
\left.\varphi\right|_{\mathcal{C}^{(n, k+n)}}\left(Q_{\lambda, \mu}^{\prime}-v\right) & =\left.\varphi\right|_{\mathcal{C}^{(n, k+n)}}\left(Q_{\lambda, \mu}^{\prime}\right)-\left.\varphi\right|_{\mathcal{C}^{(n, k+n)}}(v)+t_{\lambda, \mu} v-t_{\lambda, \mu} v \\
& =\left.\varphi\right|_{\mathcal{C}^{(n, k+n)}}\left(Q_{\lambda, \mu}^{\prime}\right)-w^{\prime}-t_{\lambda, \mu} v \\
& =t_{\lambda, \mu} Q_{\lambda, \mu}^{\prime}+w^{\prime}-w^{\prime}-t_{\lambda, \mu} v \\
& =t_{\lambda, \mu}\left(Q_{\lambda, \mu}^{\prime}-v\right) .
\end{aligned}
$$

Hence such $t_{\lambda, \mu}$ are eigenvalues of $\left.\varphi\right|_{\mathcal{C}^{(n, k+n)}}$.

Hence by induction, we have that the $t_{\lambda, \mu}$, with $|\lambda| \leq n,|\mu| \leq p$ and $|\lambda|-|\mu|=$ $n-p$, are eigenvalues of $\left.\varphi\right|_{\mathcal{C}^{(n, p)}}$.

Moreover, we have found at least $\pi(n, p)$ eigenvalues for $\left.\varphi\right|_{\mathcal{C}^{(n, p)}}$. But $\mathcal{C}^{(n, p)}$ is known to be spanned by $\pi(n, p)$ elements, so $\left.\varphi\right|_{\mathcal{C}^{(n, p)}}$ has at most $\pi(n, p)$ different eigenvalues. Hence it has exactly $\pi(n, p)$ eigenvalues each with multiplicity one.

We now state two useful corollaries.

Corollary There is a basis of $\mathcal{C}^{(n, p)}$ given by:

$$
\left\{Q_{\lambda, \mu}:|\lambda| \leq n,|\mu| \leq p,|\lambda|-|\mu|=n-p\right\}
$$

such that:

$$
\varphi\left(Q_{\lambda, \mu}\right)=t_{\lambda, \mu} Q_{\lambda, \mu} \quad \text { and } \quad \bar{\varphi}\left(Q_{\lambda, \mu}\right)=\bar{t}_{\lambda, \mu} Q_{\lambda, \mu}
$$


Corollary Every eigenvector of $\varphi$ and $\bar{\varphi}$ is a multiple of one such basis element.

Remark The eigenvalues $t_{\lambda, \mu}$ and $\bar{t}_{\lambda, \mu}$ correspond to the eigenvalues of the matrix $M$ in equation (1.1) of [3], found there only for $1 \leq k_{1}+k_{2} \leq 5$ and $k_{2} \leq k_{1}$. Chan uses the Homfly polynomial based on parameters $l$ and $m$, which are variants of $v$ and $z$. The numbers $\sqrt{m^{2}-4}$ in Chan's eigenvalues $\rho_{i}$ and $\rho_{i}^{*}$ correspond to the parameter $s$ here with $z=s-s^{-1}$, which features strongly in our eigenvalues $t_{\lambda, \mu}$ and $\bar{t}_{\lambda, \mu}$. Our use of $s$ is the feature which allows us to give simple formulae for the Gyoja-Aiston elements $Q_{\lambda}$ and to extend in principle to $Q_{\lambda, \mu}$.

Unlike the Gyoja-Aiston elements $Q_{\lambda}$ which are known and have been wellstudied, their generalisations the $Q_{\lambda, \mu}$ described in the above Corollary are not well-understood. We shall show in the following section how they can be found explicitly.

\section{The Homfly polynomials of some generalized Hopf links}

Here we apply the techniques described above to show how computation of the Homfly polynomial of some generalized Hopf links is possible.

\subsection{The Homfly polynomial of $H\left(k_{1}, k_{2} ; n, 0\right)\left(=H^{*}\left(k_{1}, k_{2} ; 0, n\right)\right)$}

Consider $H\left(k_{1}, k_{2} ; n, 0\right)$ in the skein of the annulus. Then we have

$$
H\left(k_{1}, k_{2} ; n, 0\right)=\varphi^{k_{1}}\left(\bar{\varphi}^{k_{2}}\left(A_{1}^{n}\right) .\right.
$$

Now since the maps $\varphi$ and $\bar{\varphi}$ are linear maps, we know that for the $Q_{\lambda}$,

$$
\varphi^{k_{1}}\left(\bar{\varphi}^{k_{2}}\left(Q_{\lambda}\right)\right)=t_{\lambda}^{k_{1}} \bar{t}_{\lambda}^{k_{2}} Q_{\lambda} .
$$

Also, since the $Q_{\lambda}$ are a basis or the skein $\mathcal{C}^{(n)}$, we have

$$
A_{1}^{n}=\sum_{|\lambda|=n} d_{\lambda} Q_{\lambda}
$$

for constants $d_{\lambda}$. The $d_{\lambda}$ can be calculated by several means, for example by counting the number of standard tableaux of shape $\lambda$. 
Therefore,

$$
\begin{aligned}
H\left(k_{1}, k_{2} ; n, 0\right) & =\sum_{|\lambda|=n} d_{\lambda} \varphi^{k_{1}}\left(\bar{\varphi}^{k_{2}}\left(Q_{\lambda}\right)\right) \\
& =\sum_{|\lambda|=n} d_{\lambda} t_{\lambda}^{k_{1}} \bar{t}_{\lambda}^{k_{2}} Q_{\lambda} .
\end{aligned}
$$

So evaluating in the plane (using the work of [2]), we find

$$
P\left(H\left(k_{1}, k_{2} ; n, 0\right)\right)=\sum_{|\lambda|=n} d_{\lambda} t_{\lambda}^{k_{1}} \bar{t}_{\lambda}^{k_{2}}\left(\prod_{(i, j) \in \lambda} \frac{v^{-1} s^{j-i}-v s^{i-j}}{s^{\mathrm{hl}(i, j)}-s^{-\mathrm{hl}(i, j)}}\right),
$$

where $\operatorname{hl}(i, j)$ is the hook-length of the cell $(i, j)$, in row $i$ and column $j$.

\subsection{The Homfly polynomial of $H\left(k_{1}, k_{2} ; n_{1}, n_{2}\right)$}

Consider, in a similar way to above, $H\left(k_{1}, k_{2} ; n_{1}, n_{2}\right)$ as an element of the skein $\mathcal{C}$. Then we have

$$
H\left(k_{1}, k_{2} ; n_{1}, n_{2}\right)=\varphi^{k_{1}}\left(\bar{\varphi}^{k_{2}}\left(A_{1}^{n_{1}} A_{-1}^{n_{2}}\right)\right) .
$$

Similar to the restricted case above, we have

$$
\varphi^{k_{1}}\left(\bar{\varphi}^{k_{2}}\left(Q_{\lambda, \mu}\right)\right)=t_{\lambda, \mu}^{k_{1}} \bar{t}_{\lambda, \mu}^{k_{2}} Q_{\lambda, \mu}
$$

and

$$
A_{1}^{n_{1}} A_{-1}^{n_{2}}=\sum_{\substack{|\lambda| \leq n_{2} \\|\mu| \leq n_{1} \\|\lambda|-|\mu|=n_{2}-n_{1}}} d_{\lambda, \mu} Q_{\lambda, \mu}
$$

for constants $d_{\lambda, \mu}$. These constants can be calculated in terms of appropriate $d_{\lambda}$ and $d_{\mu}$ (see previous section).

Theorem 7 [11] The numbers $d_{\lambda, \mu}$ can be found from the following formula:

$$
d_{\lambda, \mu}=m !\left(\begin{array}{c}
n_{2} \\
m
\end{array}\right)\left(\begin{array}{c}
n_{1} \\
m
\end{array}\right) d_{\lambda} d_{\mu}
$$

where $|\lambda| \leq n_{2},|\mu| \leq n_{1}$ and $m=n_{2}-|\lambda|=n_{1}-|\mu|$. 
Therefore,

$$
H\left(k_{1}, k_{2} ; n_{1}, n_{2}\right)=\sum_{\substack{|\lambda| \leq n_{2} \\|\mu| \leq n_{1} \\|\lambda|-|\mu|=n_{2}-n_{1}}} d_{\lambda, \mu} \varphi^{k_{1}}\left(\bar{\varphi}^{k_{2}}\left(Q_{\lambda, \mu}\right)\right)
$$

At present, we do not have a general closed formula for $P\left(H\left(k_{1}, k_{2} ; n_{1}, n_{2}\right)\right)$ due to lack of information about the elements $Q_{\lambda, \mu}$.

We can, however, make explicit calculations in individual cases as illustrated by the following example.

Example Consider $H\left(k_{1}, k_{2} ; 1,2\right) \in \mathcal{C}^{(2,1)}$, as shown in Figure 15.

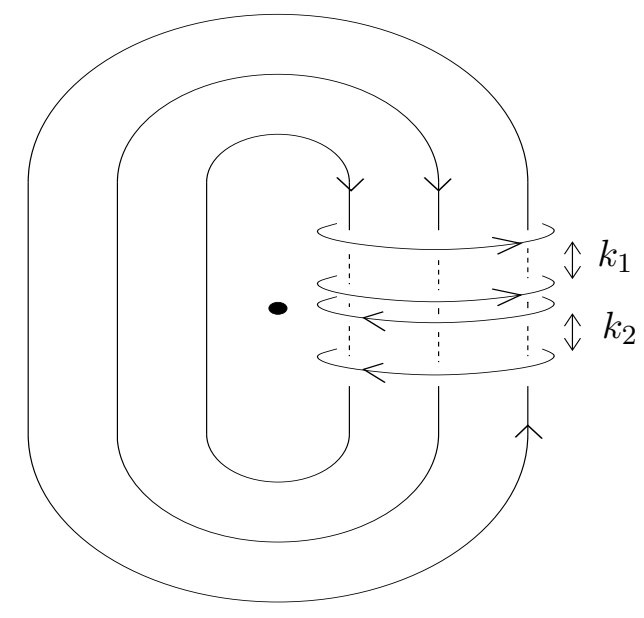

Figure 15: The link $H\left(k_{1}, k_{2} ; 1,2\right)$ in $\mathcal{C}$

Then

$$
H\left(k_{1}, k_{2} ; 1,2\right)=\varphi^{k_{1}}\left(\bar{\varphi}^{k_{2}}\left(A_{1} A_{-1}^{2}\right)\right),
$$

where, by Theorem 7 ,

$$
A_{1} A_{-1}^{2}=Q_{\square, \square}+2 Q_{\square, \emptyset}+Q_{\boxminus, \square} .
$$

However, we can also find, by using powers of trivial Gyoja-Aiston elements $Q_{\square}$, with appropriate orientation, that

$$
A_{1} A_{-1}^{2}=\left(Q_{\square}^{(-)}\right)^{2} Q_{\square}^{(+)} .
$$

Algebraic $\mathcal{E} \mathcal{G}$ Geometric Topology, Volume 2 (2002) 
Moreover, these elements are known to satisfy the Littlewood-Richardson rule for multiplication of Young diagrams ([1]), so

$$
\begin{aligned}
A_{1} A_{-1}^{2} & =\left(Q_{\square}^{(-)}+Q_{\boxminus}^{(-)}\right) Q_{\square}^{(+)} \\
& =Q_{\square}^{(-)} Q_{\square}^{(+)}+Q_{\boxminus}^{(-)} Q_{\square}^{(+)} \\
& =Q_{\square, \square}^{\prime}+Q_{\boxminus, \square}^{\prime}
\end{aligned}
$$

Now combining equations (3) and (4) with the observation that

$$
Q_{\square, \emptyset}=Q_{\square, \emptyset}^{\prime}=Q_{\square}^{(-)} Q_{\emptyset}^{(+)}
$$

and assuming symmetry under conjugation of Young diagrams, we have:

$$
\begin{aligned}
Q_{\square, \square} & =Q_{\square, \square}^{\prime}-Q_{\square, \emptyset}^{\prime}, \\
\text { and } Q_{\boxminus, \square} & =Q_{\boxminus, \square}^{\prime}-Q_{\square, \emptyset}^{\prime} .
\end{aligned}
$$

Hence, evaluating in the plane, we find,

$$
\begin{aligned}
P\left(H\left(k_{1}, k_{2} ; 1,2\right)\right)= & P\left(\varphi^{k_{1}}\left(\bar{\varphi}^{k_{2}}\left(A_{1} A_{-1}^{2}\right)\right)\right) \\
= & t_{\square, \square}^{k_{1}} \bar{t}_{\square, \square}^{k_{2}} P\left(Q_{\square, \square}\right) \\
& +2 t_{\square, \emptyset}^{k_{1}} \bar{t}_{\square, \emptyset}^{k_{2}} P\left(Q_{\square, \emptyset}\right)+t_{\boxminus, \square}^{k_{1}} \bar{t}_{\boxminus, \square}^{k_{2}} P\left(Q_{\boxminus, \square}\right) \\
= & t_{\square, \square}^{k_{1}} \bar{t}_{\square, \square}^{k_{2}}\left(P\left(Q_{\square, \square}^{\prime}\right)-P\left(Q_{\square, \emptyset}^{\prime}\right)\right) \\
& \left.+2 t_{\square, \emptyset}^{k_{1}, \bar{t}^{k_{2}} P\left(Q_{\square, \emptyset}^{\prime}\right.}\right)+t_{\boxminus, \square}^{k_{1}} \bar{t}_{\boxminus, \square}^{k_{2}}\left(P\left(Q_{\boxminus, \square}^{\prime}-P\left(Q_{\square, \emptyset}^{\prime}\right)\right)\right. \\
= & t_{\square, \square}^{k_{1}} \bar{t}_{\square, \square}^{k_{2}} P\left(Q_{\square, \square}^{\prime}\right) \\
& +\left(2 t_{\square, \emptyset}^{k_{1}} \bar{t}_{\square, \emptyset}^{k_{2}}-t_{\square, \square}^{k_{1}} \bar{t}_{\square, \square}^{k_{2}}-t_{\boxminus, \square}^{k_{1}} \bar{t}_{\square, \square}^{k_{2}}\right) P\left(Q_{\square, \emptyset}^{\prime}\right) \\
& +t_{\square, \square}^{k_{1}} \bar{t}_{\square, \square}^{k_{2}} P\left(Q_{\square, \square}^{\prime}\right)
\end{aligned}
$$

From the definition of the $Q_{\lambda, \mu}^{\prime}$, we can now use the results in [2] to find $P\left(Q_{\square, \emptyset}^{\prime}\right), P\left(Q_{\square, \square}^{\prime}\right)$ and $P\left(Q_{\boxminus, \square}^{\prime}\right)$. We have:

$$
\begin{aligned}
P\left(Q_{\square, \emptyset}^{\prime}\right) & =\frac{v^{-1}-v}{s-s^{-1}}, \\
P\left(Q_{\square, \square}^{\prime}\right) & =\left(\frac{v^{-1}-v}{s^{2}-s^{-2}}\right)\left(\frac{v^{-1} s-v s^{-1}}{s-s^{-1}}\right)\left(\frac{v^{-1}-v}{s-s^{-1}}\right), \\
\text { and } P\left(Q_{\square, \square}^{\prime}\right) & =\left(\frac{v^{-1}-v}{s^{2}-s^{-2}}\right)\left(\frac{v^{-1} s^{-1}-v s}{s-s^{-1}}\right)\left(\frac{v^{-1}-v}{s-s^{-1}}\right) .
\end{aligned}
$$

Algebraic $\mathcal{E} \mathcal{G}$ Geometric Topology, Volume 2 (2002) 
Then using Proposition 4 we find:

$$
\begin{aligned}
t_{\square, \emptyset} & =-v\left(s-s^{-1}\right)+\delta, \\
t_{\square, \square} & =\left(s-s^{-1}\right)\left(-v\left(1+s^{-2}\right)+v^{-1}\right)+\delta, \\
t_{\boxminus, \square} & =\left(s-s^{-1}\right)\left(-v\left(1+s^{2}\right)+v^{-1}\right)+\delta,
\end{aligned}
$$

and

$$
\begin{aligned}
\bar{t}_{\square, \emptyset} & =v^{-1}\left(s-s^{-1}\right)+\delta, \\
\bar{t}_{\square, \square} & =\left(s-s^{-1}\right)\left(v^{-1}\left(1+s^{2}\right)-v\right)+\delta, \\
\bar{t}_{\boxminus, \square} & =\left(s-s^{-1}\right)\left(v^{-1}\left(1+s^{-2}\right)-v\right)+\delta .
\end{aligned}
$$

Substitution of these values into equation (5) then gives $P\left(H\left(k_{1}, k_{2} ; 1,2\right)\right)$ immediately.

\subsection{A final remark}

We can in principle write any given element of the skein $X \in \mathcal{C}$ as a linear combination of the basis elements $Q_{\lambda, \mu}$. Therefore, one can find $\varphi(X)$ and $\bar{\varphi}(X)$, and hence readily evaluate the Homfly polynomial of $H\left(k_{1}, k_{2} ; X\right):=$ $H_{+}\left(X, A_{1}^{k_{1}} A_{-1}^{k_{2}}\right)$. The special case $X=A_{1}^{n_{1}} A_{-1}^{n_{2}}$ gives $H\left(k_{1}, k_{2} ; n_{1}, n_{2}\right)$.

\section{Acknowledgments}

The second author was supported by EPSRC grant 99801479 .

\section{References}

[1] A K Aiston, Skein theoretic idempotents of Hecke algebras and quantum group invariants, Ph.D. thesis, University of Liverpool (1996)

[2] A K Aiston, H R Morton, Idempotents of Hecke algebras of type A, J. Knot Theory Ramif. 7 (1998) 463-487

[3] T-H Chan, HOMFLY polynomial of some generalized Hopf links, J. Knot Theory Ramif. 9 (2000) 865-883

[4] A Gyoja, A q-analogue of Young symmetrizers, Osaka J. Math. 23 (1986) $841-852$

[5] R J Hadji, Knots, tangles and algebras, M.Sc. Dissertation, University of Liverpool (1999) 
[6] S G Lukac, Homfly skeins and the Hopf link, Ph.D. thesis, University of Liverpool (2001)

[7] HR Morton, Skein theory and the Murphy operators, preprint, arxiv: math.GT/0102098, to appear in "Proceedings of Knots 2000, Korea"

[8] H R Morton, Invariants of links and 3-manifolds from skein theory and from quantum groups, from: "Proceedings of the NATO Summer Institute in Erzurum 1992, NATO ASI Series C 399", (M Bozhüyük, editor), Kluwer (1993) 107-156

[9] H R Morton, P Traczyk, Knots and algebras, from: "Contribuciones Mathematicas en homenaje al profesor D. Antonio Plans Sanz de Bremond", (E Martin-Peinador, A Rodez Usan, editors), University of Zaragoza (1990) 201220

[10] H R Morton, A J Wassermann, String algebras and oriented tangles, personal communication of notes from the first author

[11] J R Stembridge, Rational tableaux and the tensor algebra of $g l_{n}$, J. Combin. Theory 46 (1987) 79-120

[12] V G Turaev, The Conway and Kauffman modules of the solid torus with an appendix on the operator invariants of tangles, Progress in Knot Theory and Related Topics 56 (1997) 90-102

Department of Mathematical Sciences, University of Liverpool

Peach Street, Liverpool, L69 3ZL, UK

Email: morton@liv.ac.uk, rhadji@liv.ac.uk

URL: http://www.liv.ac.uk/ su14/knotgroup.html

Received: 25 June 2001 Revised: 11 January 2002 